\title{
Wake Measurement Downstream of a Hybrid Wing Body Model with Blown Flaps
}

\author{
John C. Lin," Gregory S. Jones," and Brian G. Allan* \\ NASA Langley Research Center, Hampton, Virginia, 23681, USA \\ john.c.lin@nasa.gov \\ Bryan W. Westra ${ }^{\dagger}$ and Scott W. Collins ${ }^{\ddagger}$ \\ Northrop Grumman Corporation, El Segundo, California, 90245, USA \\ Cale H. Zeune ${ }^{\S}$ \\ Air Force Research Laboratory, Wright-Patterson AFB, Ohio, 45433, USA
}

\begin{abstract}
Flow-field measurements were obtained in the wake of a full-span Hybrid Wing Body model with internally blown flaps. The test was performed at the NASA Langley $14 \times 22$ Foot Subsonic Tunnel at low speeds. Off-body measurements were obtained with a 7-hole probe rake survey system. Three model configurations were investigated. At $0^{\circ}$ angle of attack the surveys were completed with $0^{\circ}$ and $60^{\circ}$ flap deflections. At $10^{\circ}$ angle of attack the wake surveys were completed with a slat and a $60^{\circ}$ flap deflection. The 7 -hole probe results further quantified two known swirling regions (downstream of the outboard flap edge and the inboard/outboard flap juncture) for the $60^{\circ}$ flap cases with blowing. Flowfield results and the general trends are very similar for the two blowing cases at nozzle pressure ratios of 1.37 and 1.56. High downwash velocities correlated with the enhanced lift for the $60^{\circ}$ flap cases with blowing. Jet-induced effects are the largest at the most inboard station for all (three) velocity components due in part to the larger inboard slot height. The experimental data are being used to improve computational tools for high-lift wings with integrated powered-lift technologies.
\end{abstract}

\section{INTRODUCTION}

NASA's Subsonic Fixed Wing Project is pursuing technologies and the validation of tools to enable cruise-efficient (CE), short take-off and landing (STOL) aircraft for civilian purposes. The enabling tools and technologies are dual use in that they are applicable for both military and civilian missions. As the result of a research and development collaboration between NASA Langley Research Center (LaRC), the Air Force Research Laboratory (AFRL), and the Northrop Grumman Corporation (Northrop Grumman), an all-wing aircraft with a blown-flap high-lift system, or the Hybrid Wing Body (HWB) concept, has emerged as a candidate to provide databases for the development of computational fluid dynamics (CFD) tools for the CE STOL efforts.

The inherent benefits of all-wing designs are well known in terms of cruise efficiency compared to conventional aircraft $[1,2]$. The HWB aircraft concept studied here leverages planform area while managing the risk in achieving the lift required for STOL operations. That is to say, if a low to moderate wing loading is chosen for conventional take-off and landing distances, then the amount of additional lift required for STOL objectives is minimized. The centerbody of the current HWB

\footnotetext{
* Aerospace Engineer, Flow Physics and Control Branch, MS 170

${ }^{\dagger}$ Manager, Propulsion System Design and Integration, MS 9V14/W6

${ }^{\star}$ Director, Future Tactical Systems, MS XE 40/W6

$\S$ Aerospace Engineer, AFRL/RBAA
} 
concept produces one-third of the vehicle's total lift while the wings with internally blown flaps produce the remaining two-thirds [3].

The joint NASA/AFRL/Northrop Grumman research efforts were conducted at the NASA Langley 14 x 22 Foot Subsonic Tunnel to examine a HWB model's high-lift performance. Part of these joint efforts involved a flow physics investigation using a 7-hole probe wake rake system to measure the off-body flow fields downstream of the model. The investigation was conducted to assess the complex flow interactions in the near wake of the blown flaps as well as to provide a flow-field database to the CFD community to evaluate computations. These data are being used in the development and validation of CFD tools to advance powered-lift/circulation-control technologies by contributing to the "Subsystem Cases" of AIAA's four-step code validation process adopted by NASA [4]. The process includes experiments documenting Unit Problems, Benchmark Cases, Subsystem Cases, and Complete System [5]. Collins, et al. [3] reported the incremental lift and surface pressure data of the HWB model. In this paper we will focus only on the results of the wake measurements.

\section{FACILITY DESCRIPTION}

The NASA Langley $14 \times 22$ Foot Subsonic Tunnel $(14 \times 22)$ is an atmospheric, closed return tunnel with a test section $\mathrm{m} 4.42 \mathrm{~m}(14.5 \mathrm{ft})$ high, $6.63 \mathrm{~m}(21.75 \mathrm{ft})$ wide, and $15.24 \mathrm{~m}(50 \mathrm{ft})$ long, a maximum freestream velocity of $103 \mathrm{~m} / \mathrm{s}(338 \mathrm{ft} / \mathrm{s})$, and a dynamic pressure $(q)$ of $6.89 \mathrm{kPa}(144 \mathrm{psf})$. The Reynolds number $(\mathrm{Re})$ ranges from 0 to $7.2 \times 10^{6}$ per meter $\left(2.2 \times 10^{6}\right.$ per foot). Test section airflow is driven by a $12.19 \mathrm{~m}(40 \mathrm{ft})$ diameter, 9-bladed fan powered by a 12,000-hp solid-state converter with synchronous motor. The tunnel has a set of flow control vanes to maintain control of the speed for low-speed testing. The closed test section configuration was used for the current test, and the configuration produced relatively uniform flow with a velocity fluctuation of 0.1 percent or less [6]. The current flow-field investigation was conducted at a freestream Mach number $\left(\mathrm{M}_{\infty}\right)$ of 0.143 , a $q$ of $1.44 \mathrm{kPa}(30 \mathrm{psf})$, and a of Re of $3.1 \times 10^{6}$ per meter $\left(9.6 \times 10^{5}\right.$ per foot $)$.

\section{MODEL DESCRIPTION}

The test article was a full-span sting-mounted model (see Fig. 1) with facility supplied high-pressure air for its internally blown flaps. The model was developed by Northrop Grumman for AFRL to advance the state-of-the-art of integrated high-lift and control technologies. The HWB model has a span of $2.44 \mathrm{~m}(8 \mathrm{ft})$, a length of $1.43 \mathrm{~m}(4.7 \mathrm{ft})$, and a mean aerodynamic chord of $0.84 \mathrm{~m}(2.75 \mathrm{ft})$. Trailing-edge flap deflections of $0^{\circ}$ and $60^{\circ}$ (downward) were used for the current investigation. Wake surveys were conducted with and without the baseline slat that has a $60^{\circ}$ downward deflection. CFD runs were used to determine the transition grit size and location on the model. The analysis concluded that No. 60 grit $(0.29 \mathrm{~mm})$ located $25.4 \mathrm{~mm}(1 \mathrm{inch})$ normal from the leading edge on the inboard section of the model would adequately force transition. The outboard section of the wing was not gritted since the presence of the slat will force natural transition. The same grit size and location was applied on the lower surface.

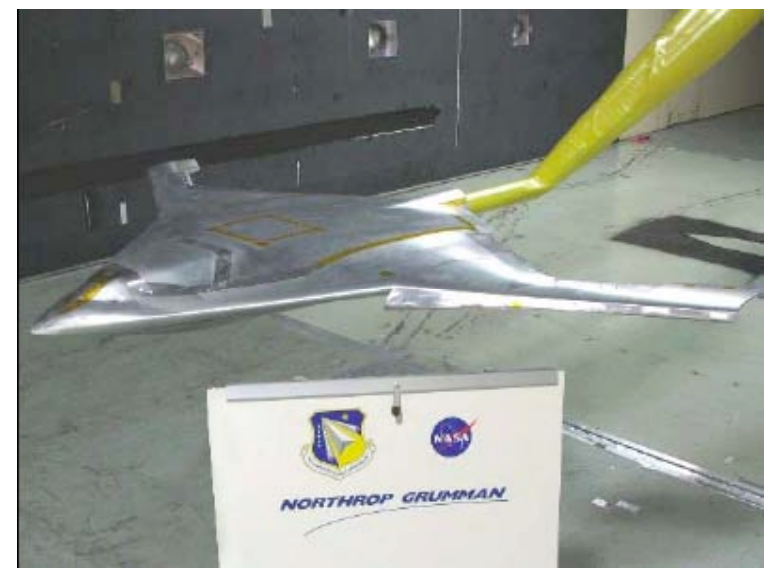

Figure 1. HWB Model in $14 \times 22$. 
The model was mounted on a flow-through bent sting. The Langley balance 1621 was used to measure forces and moments on the model. Because of the high-pressure air needs, the model balance was used with the "air sting" support system to provide a non-metric to metric high-pressure air balance crossover. Figure 2 shows the metric components of the model support system, which included air sting coil, accumulator, model, balance block, and balance.
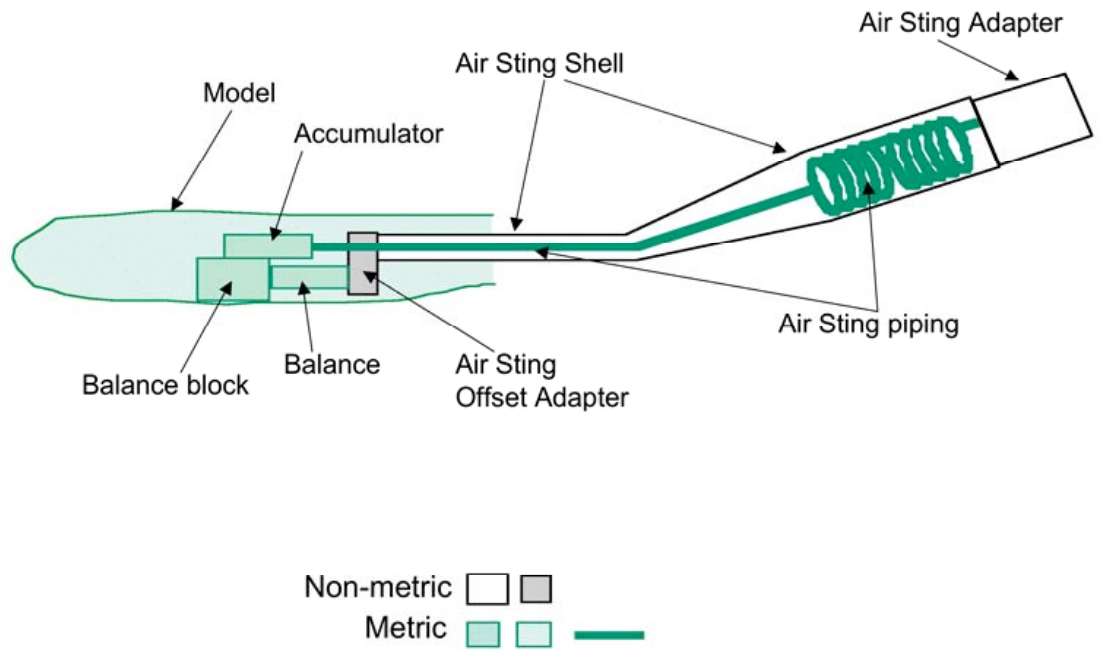

Figure 2. Air sting support components.

High-pressure air at approximately $1.59 \times 10^{3} \mathrm{kPa}(230 \mathrm{psig})$ enters the model through a tube in the hollow support sting. The accumulator distributes air to high aspect-ratio slots forward of the wing flap surfaces. There are four blowing slots, left-hand (LH) and right-hand (RH) inboard flaps as well as the LH and RH outboard flaps. Four distribution valves inside the model were used to control the nozzle pressure ratio (NPR) at each slot, where NPR is defined as the ratio of total pressure over freestream static pressure. NPR ratios of 1.0 (non-blowing case), 1.37, and 1.56 were examined in the current study.

The outboard flap had a constant-height slot $\left(\mathrm{h}_{\mathrm{OB}}\right)$. The inboard blowing slot was linearly tapered in height (h), starting with $\mathrm{h}=4 \mathrm{x} \mathrm{h}_{\mathrm{OB}}$ at the inboard edge and transitioning to $\mathrm{h}_{\mathrm{OB}}$ at the outboard edge. Porous choke plates are used to manage flow at each blowing slot. The choke plates are designed to create a large pressure drop from the facility air supply to the set total pressure at the slot nozzle. The choke plates are designed to provide uniform, total pressure across the entire slot width for both the tapered inboard slot and the constant height outboard slot. The Mach number is sonic (M $=1$ ) for exit NPRs above approximately 1.03. Therefore, because the slot flow rate varies linearly with pressure, the choke plates, once calibrated, can be used as accurate flow meters for each slot. The summation of all four calculated choke plate flows was also compared against the facility flow meter. Typical agreement was within $+/-2 \%$ for the test.

\section{7-HOLE PROBE FLOW FIELD SURVEY}

Aerodynamicists have employed a variety of different types of multi-hole probes for flow diagnostics in complex flow fields. The 7-hole probe technique used in this study involves a standard, welldocumented method of defining a set of non-dimensional coefficients based on the location of the maximum of the seven pressure readings from the probe [7-12].

The 7-hole probes used in the current investigation were fabricated and calibrated by Aeroprobe Corporation. The 7-hole probes provide the three components of velocity for flow angles as high as $75^{\circ}$ with respect to the probe axis. These probes are rugged, and once calibrated require minimal maintenance to achieve an accuracy that is typically within $1 \%$ of the measured flow speed and $0.5^{\circ}$ in 
flow angles. These accuracies are also dependent on the pressure transducer accuracy, typically less than $0.1 \%$ of full-scale.

The current 7-hole probe system is made up of a rake head holding eight 7-hole probes with a vertical spacing of one inch between probe tips as well as a positioning system traversing $1001 \mathrm{~mm}$ (39.4 inches) in the horizontal spanwise (y) direction and $305 \mathrm{~mm}$ (12 inches) in the vertical (z) direction, as shown in Fig. 3. The 7-hole probe measurements were obtained in the wake at selected horizontal streamwise (x) locations downstream of the left wing. The U-, V-, and W-velocity components are measured in the tunnel coordinate system, corresponding to velocities in the $\mathrm{x}, \mathrm{y}$, and $\mathrm{z}$ direction, respectively. Test results are to be presented in the non-dimensional coordinate system $\mathrm{X}$, $\mathrm{Y}$, and $\mathrm{Z}$, where they correspond to $\mathrm{x}, \mathrm{y}$, and $\mathrm{z}$ respectively normalized by a length scale roughly equal to half the model span $(1.27 \mathrm{~m})$.

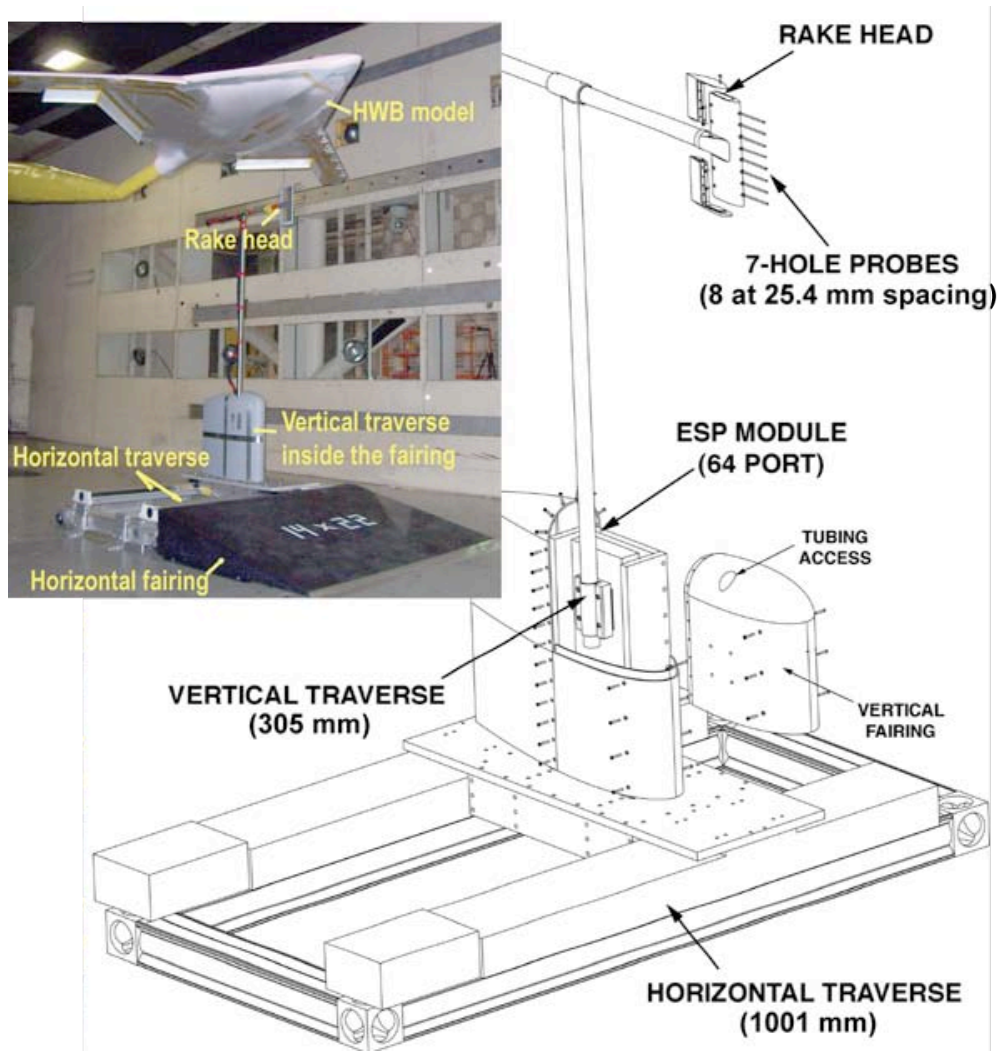

Figure 3. Wake rake survey system.

\section{RESULTS AND DISCUSSION}

The wakes from three configurations of the HWB model with blown flaps were investigated with the 7-hole probes. The three configurations were: A) $60^{\circ}$ flap deflection, leading edge slat-on, and $\alpha$ (angle of attack) $=10^{\circ}$, B) $0^{\circ}$ flap deflection at $\alpha=0^{\circ}$, and C) $60^{\circ}$ flap deflection at $\alpha=0^{\circ}$. All configurations were at $0^{\circ}$ yaw angle. Case $\mathrm{A}$ has the most resolution in the survey grid and included several survey stations in the $\mathrm{X}$ direction. Because of the time constraint towards the end of the test, the latter two cases, B and C, have fewer points in the grid than A. Flow features observed from the wake survey are discussed in the following sections.

\subsection{Case A: $60^{\circ}$ Flap and Slat-On at $\alpha=10^{\circ}$}

Once flow control is established, the flow features and aerodynamic performance were found to be very similar between the two blowing cases (NPR $=1.37$ and 1.56). Since the lower blowing case has an obvious advantage in pneumatic supply system requirements, only NPR $=1.37$ results are presented 
in this section and are considered as representative of the blown flap case for this model. The flowfield data at $X=1.08$ for the $60^{\circ}$ flap and baseline slat-on model configuration at $\alpha=10^{\circ}$ are shown in Fig. 4, where $X=1.08$ corresponds approximately to the trailing edge apex of the outboard wing (see the left sketch on top of Fig. 6). The data are presented in terms of streamwise mean velocity (U) contours and velocity vectors in the Y-Z (cross-flow) plane for NPR of 1.0 (non-blowing) and 1.37. White boxes in the middle of the vortex or wake indicate high angularity in the local flows. Flows near the core of a wake vortex or near the off-body re-circulating regions could have flow angles greater than $75^{\circ}$ with respect to the probe axis, which are outside the measuring capability of our 7hole probes.

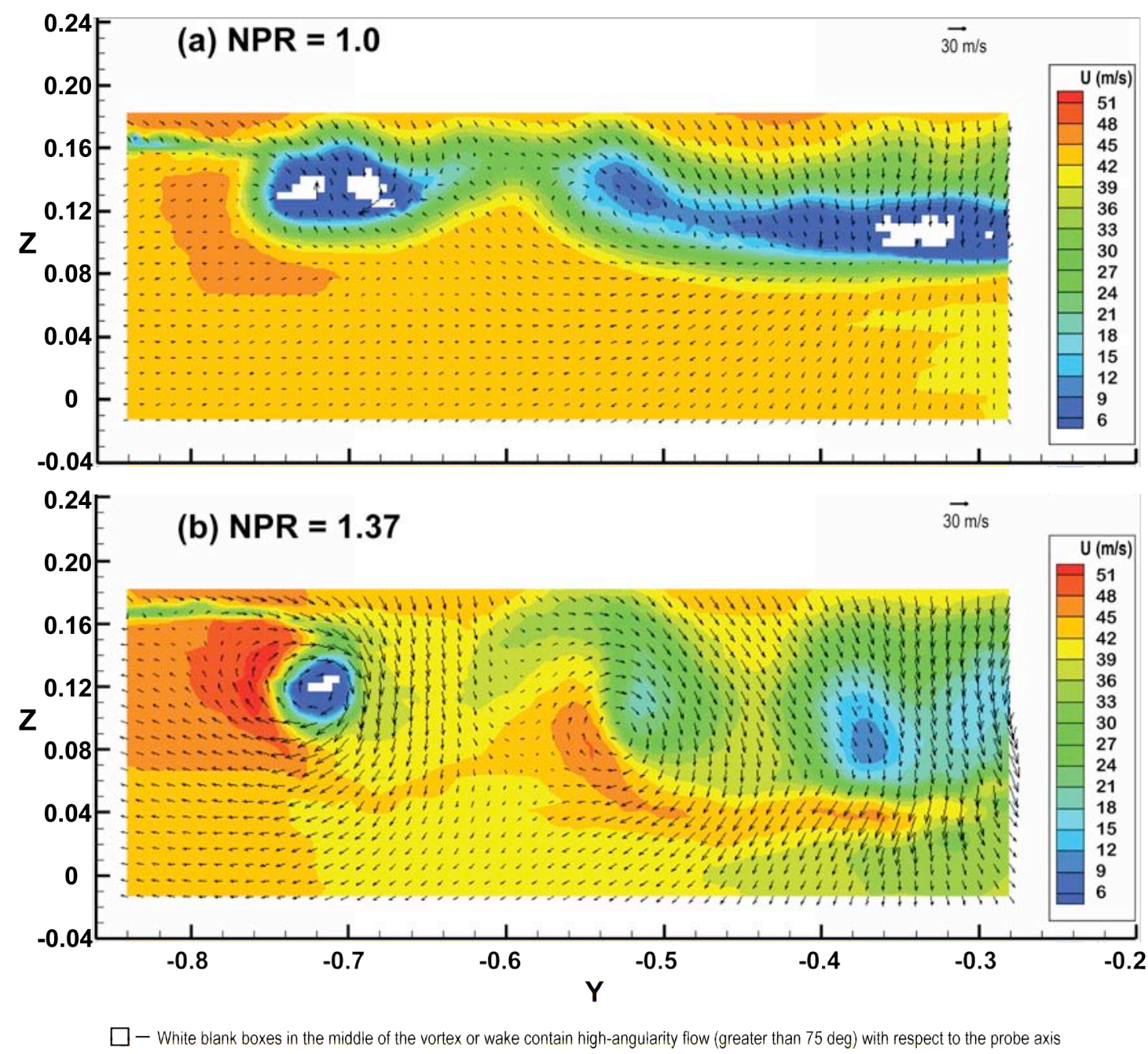

Figure 4. U-velocity contours and velocity vectors in $\mathrm{Y}-\mathrm{Z}$ plane at $\mathrm{X}=1.08\left(\mathrm{M}_{\infty}=0.143,60^{\circ}\right.$ flap and slat-on, $\alpha=10^{\circ}$ ). (a) NPR $=1.0$; and (b) NPR $=1.37$.

For NPR $=1.0$ (Fig. 4 (a)), the large velocity deficit in the U-velocity contours between $Y=-0.66$ and -0.74 corresponds to the wake vortex associated with the outboard edge of the outboard flap. For the inboard flap, the velocity deficit is concentrated downstream of its inboard half and is considerably smaller towards the juncture between the inboard and outboard flaps $(\mathrm{Y}=-0.57)$. This may be due to the inboard wing trailing-edge forward-sweep increasing the distance from the flap to the survey plane. There also appears to be a weak juncture-induced swirling slightly towards the inboard side. 
For NPR $=1.37$ (Fig. 4 (b)), the velocity vectors clearly show two swirling regions in the Y-Z plane. The increased lift (loading) and the effects of blowing jets significantly enhanced the strength of the (stronger) flap-edge-induced outboard vortex and the (weaker) juncture-induced swirling. The weaker swirling has significantly less velocity deficit in its core than the stronger (outboard) vortex. When viewing towards the upstream direction, the swirling motion is clockwise. The outer edge of a third vortex caused by the inboard edge of the inboard flap can be observed at the right side of the plots, but the main part of the vortex was just outside of the survey area. The expected inboard flap edge vortex has a counter-clockwise rotation when viewing upstream. The swirling vortices of the blowing case segmented the wake into several three-dimensional structures.

Flow-field results for the blowing case are presented in Fig. 5 in terms of Y-Z plane contours at X $=1.08$ for the velocity magnitude (Vmag), the ratio of probe total pressure over freestream total pressure (Pt/Pto), and the vorticity. The results indicate concentrated regions of high Vmag and $\mathrm{Pt} / \mathrm{Pto}$ located at $\mathrm{Z} \approx 0.04$ near the inboard flap (see Figs. 5 (a) and 5 (b)). These high Vmag and Pt/Pto regions result from the significant effect of momentum addition from the inboard blowing jet. The Uvelocity component of Fig. 4 is smaller compared to the Vmag of Fig. 5 (a) in regions above and below the inboard jet sheet. This indicates that a significant amount of downwash velocity exists, as expected for the high-lift system. The high degree of flow turning is also seen in the vector field plot. The vorticity plot (Fig. 5 (c)) confirms the two regions of concentrated negative vorticity associated with the two known swirling regions (downstream of the outboard flap edge and downstream of the inboard/outboard flap juncture). Two small pockets of concentrated positive vorticity, which might be associated with the inboard flap-edge-induced vortex, are also observed near the bottom right region of the plot.

For NPR $=1.0$ and 1.37, the U-, V-, and W-velocity distributions along the Z-axis (vertical) are shown in Figs. 6 and 7, respectively. The Part (a) of each figure shows the U-velocity profile, while Parts (b) and (c) show the V- and W-velocity profiles, respectively. The plots along the three rows show the three spanwise stations of $Y=-0.42,-0.57$, and -0.65 , while the plots along the four columns show the four X stations of $0.99,1.08,1.16$, and 1.24. The sketches at the top of each figure show the location of these $(\mathrm{X}, \mathrm{Y})$ stations with respect to the model, where the blue lines also indicate the extent of the survey in the vertical direction. According to the defined coordinate system, positive V-velocity represents inward flow towards the fuselage and negative represents outward flow towards the wing tip; similarly, positive W-velocity represents upward flow and negative represents downward flow. For clarity, the freestream value $(\sim 48 \mathrm{~m} / \mathrm{s})$ for the U-velocity and the zeros for V- and W-velocity were marked in these plots as references.

The U-, V-, and W-velocity profiles are distinctive from one another at each measuring location. Figures 6 (a) and 7 (a) show that the U-velocity deficits in the wake were largest nearest to the model $(\mathrm{X}=0.99)$ and grew smaller, as expected, as the wake moved farther away. For both blowing (NPR = $1.37)$ and non-blowing $(\mathrm{NPR}=1.0)$ cases, the $\mathrm{U}$-velocity deficits decreased more rapidly downstream of the outboard flap ( $\mathrm{Y}=-0.65)$ when compared to those of the juncture $(\mathrm{Y}=-0.57)$ or inboard flap ( $\mathrm{Y}$ $=-0.42$ ) stations. Perhaps this is due to the enhanced mixing produced by the swirling vortices observed in Figs. 4 and 5, where the blowing case showed significant spreading of the wakes in the Zdirection and distinctive "S-shaped" U-velocity profiles (see Figure 7 (a)). The bottom half of the Sshaped profile is due to the momentum addition of the blowing jets where their effect is strongest nearest to the model.

Figures 6 (b) and 7 (b) show that the V-velocities are generally positive (inward flow) in the top portion of the surveys, with negative velocities (outward flow) in the bottom portion. The magnitudes of the V-velocities in both directions are generally larger for the blowing case due to the stronger lift and vortices present in the flow field, which enhanced the inward flow on the top and outward flow on the bottom. The boundaries between the inward and outward flows are also generally lower for the blowing case.

The W-velocities are mostly negative (downwash flows), as shown in Figs. 6 (c) and 7 (c). Small regions of up-wash flows are noted near the middle portion of the survey $(\mathrm{Z} \approx 0.08$ to 0.14$)$ for the non-blowing case and downstream of the juncture $(\mathrm{Y}=-0.57)$ station for the blowing case. The high negative values are another indication of enhanced lift due to the blown flap. The downwash magnitudes are largest downstream of the inboard and outboard stations nearest to the model for the 
blowing case. In fact, as seen in Fig. 7 (c) at $X=0.99$ and $Y=-0.42$, the magnitude of the downwash velocity exceeds the value of the freestream velocity.
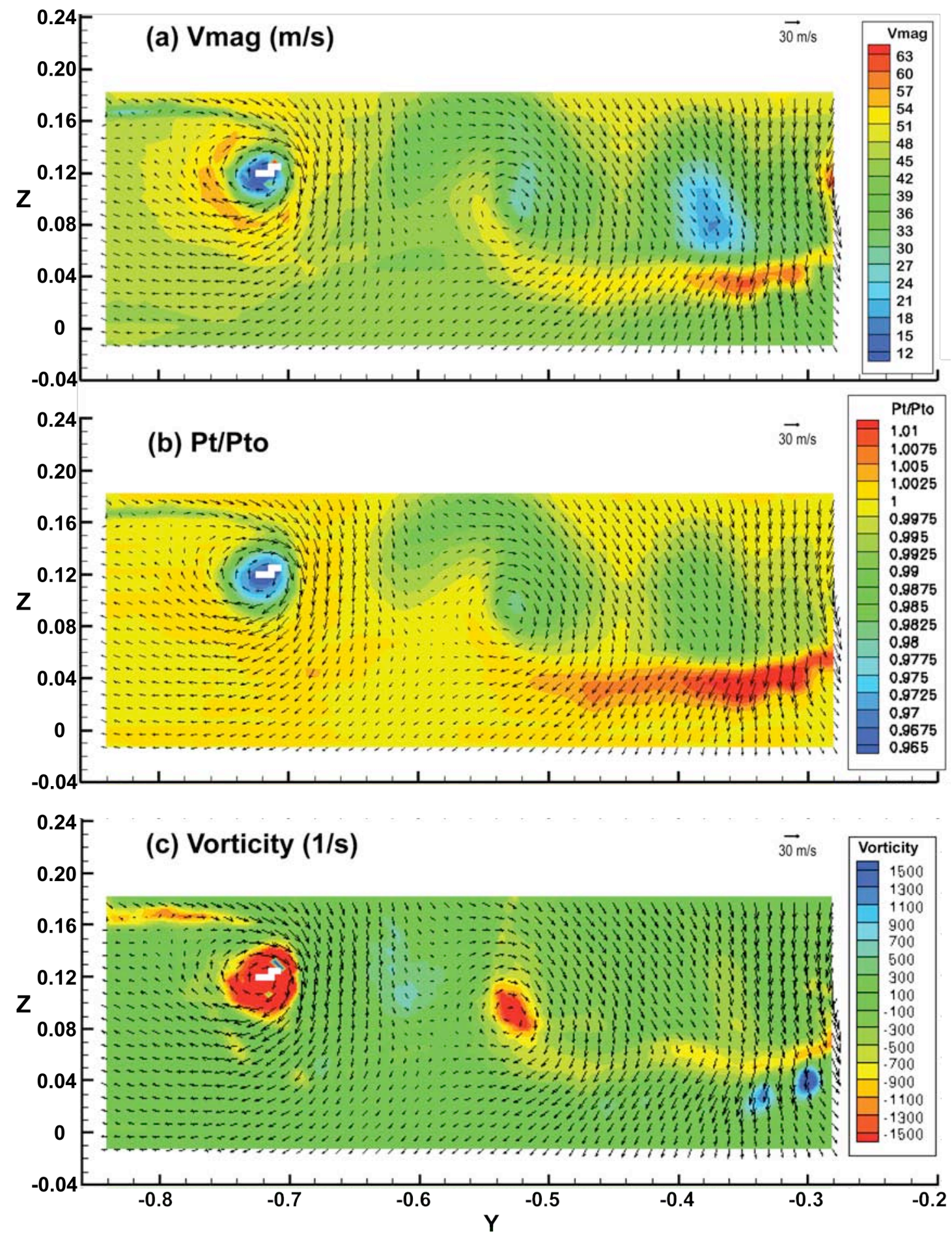

Figure 5. Vmag, $P t / P t o$, and vorticity contours in $Y-Z$ plane at $X=1.08\left(M_{\infty}=0.143,60^{\circ}\right.$ flap and slat-on, $\alpha=10^{\circ}$, NPR $=1.37$ ). (a) Vmag; (b) Pt/Pto; and (c) vorticity. 

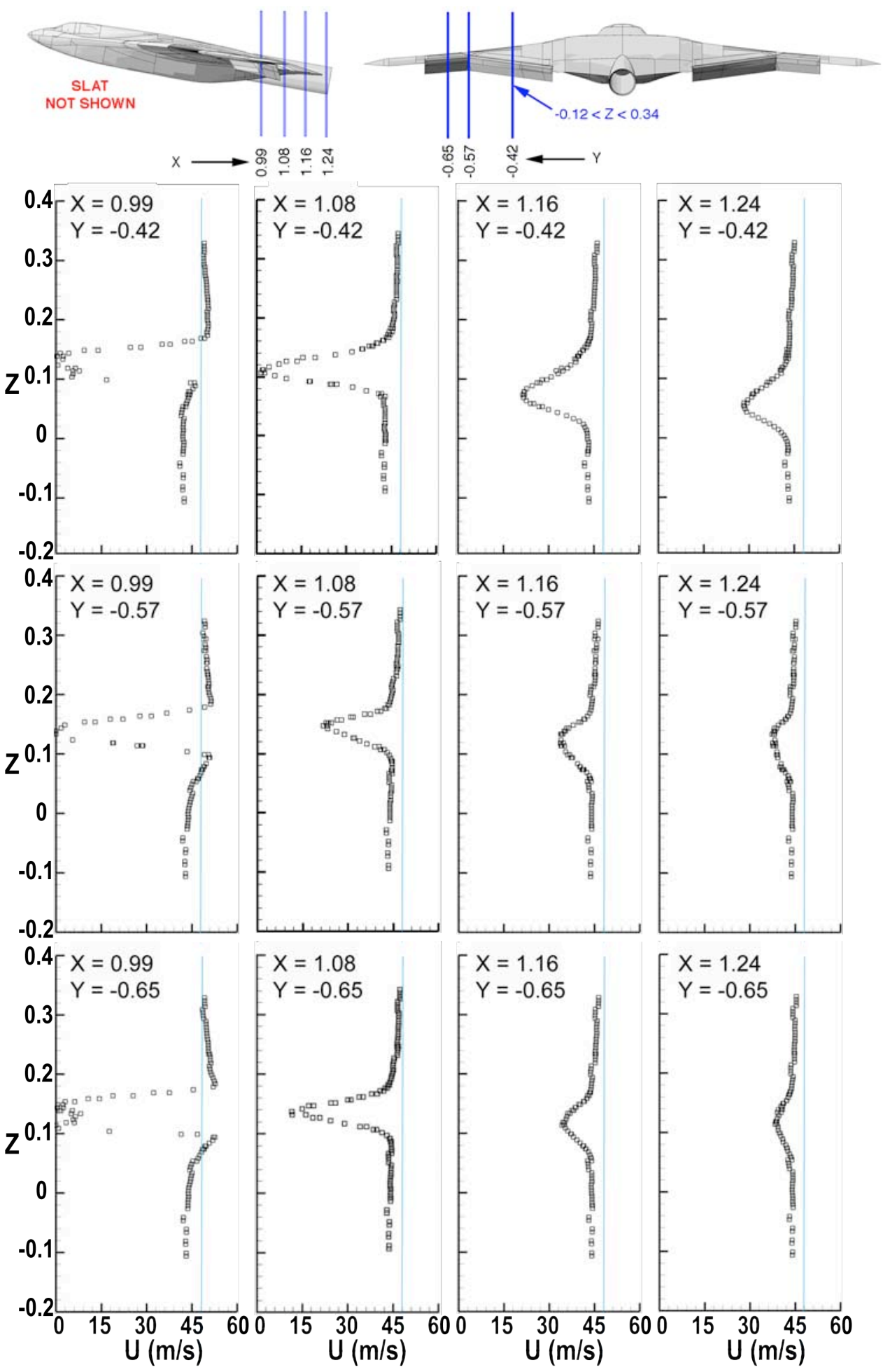

(a) U-velocity profiles

Figure 6. U-, $\mathrm{V}$-, and $\mathrm{W}$-velocity profiles for model with $60^{\circ}$ flap and slat-on at NPR $=1.0\left(\mathrm{M}_{\infty}\right.$ $=0.143, \alpha=10^{\circ} ; X=0.99,1.08,1.16$, and 1.24; $Y=-0.42,-0.57$, and -0.65 ). (a) U-velocity profiles; (b) $\mathrm{V}$-velocity profiles; and (c) W-velocity profiles. 

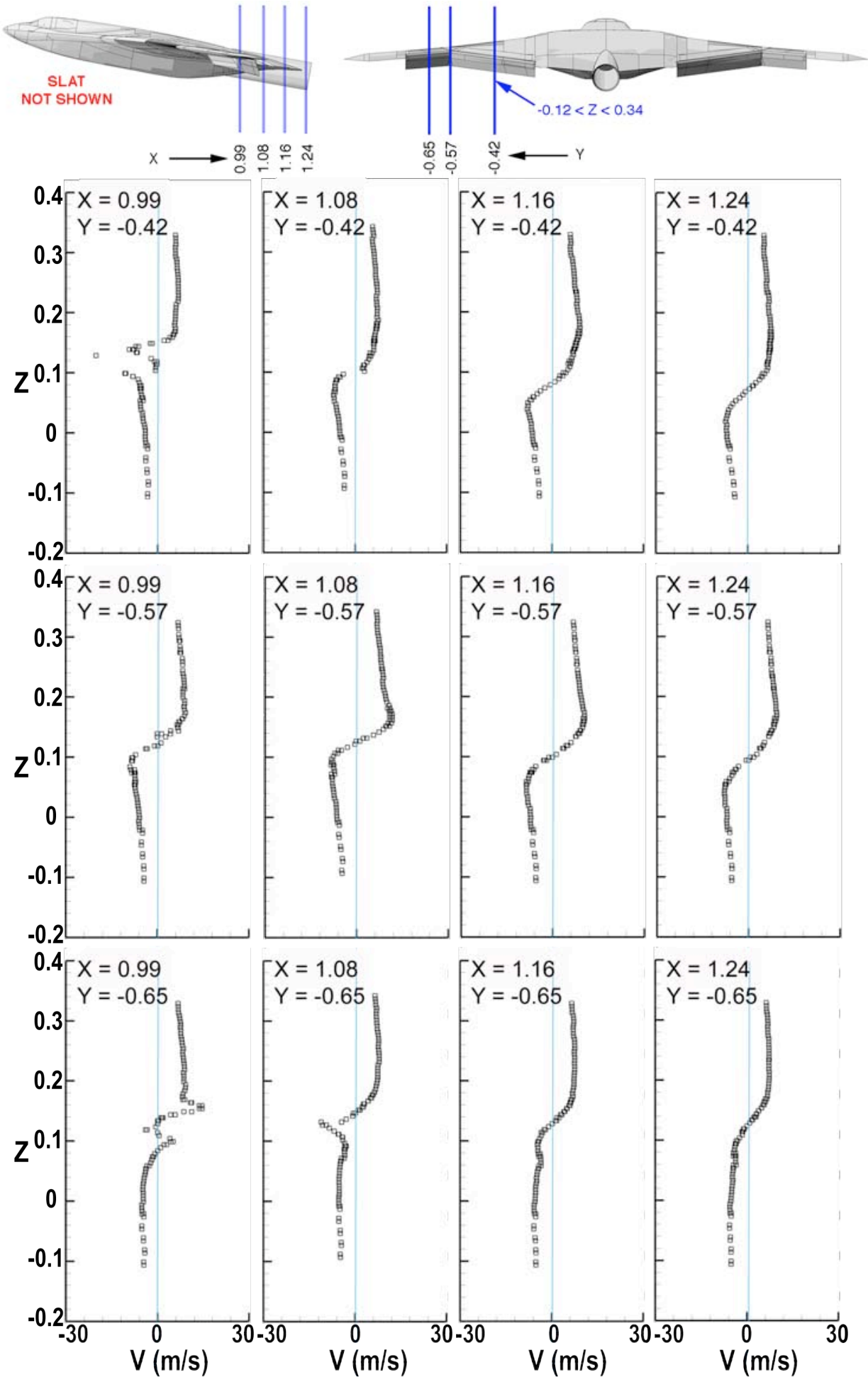

(b) V-velocity profiles (+ represents inward flow, - represents outward flow)

Figure 6. Continued $(N P R=1.0)$. 


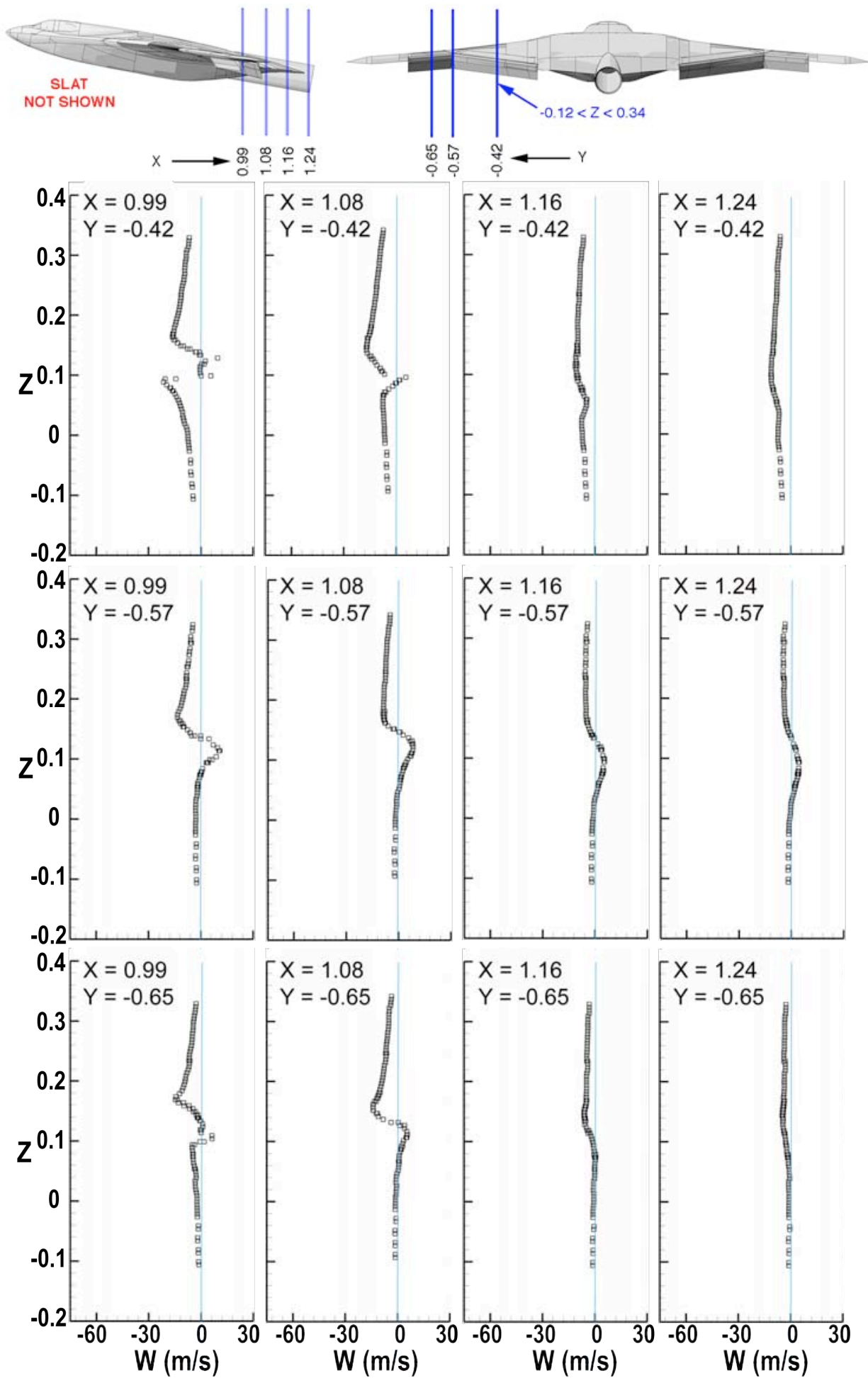

(c) W-velocity profiles (+ represents upward flow, - represents downward flow)

Figure 6. Concluded $(\mathrm{NPR}=1.0)$. 


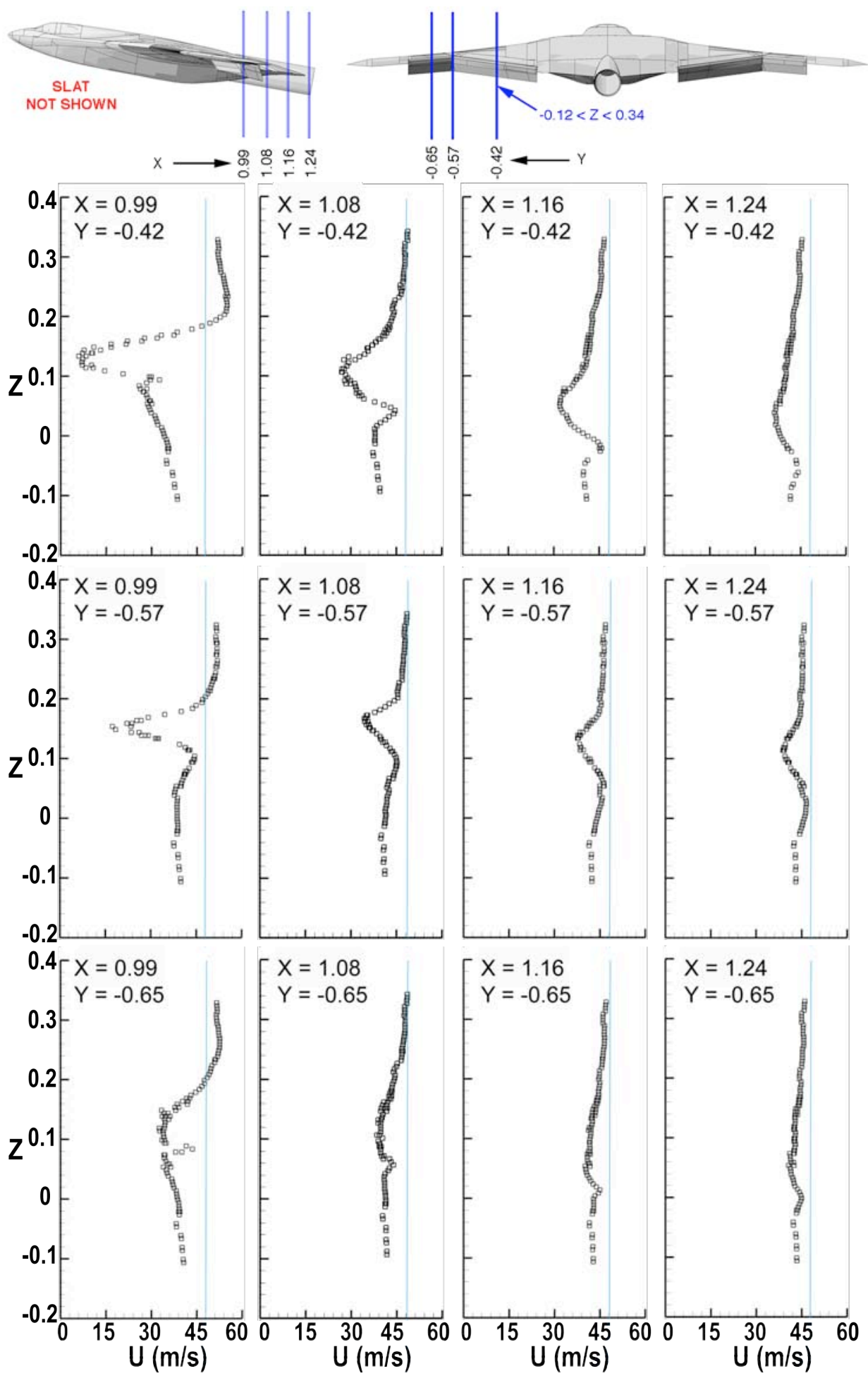

(a) U-velocity profiles

Figure 7. U-, V-, and $\mathrm{W}$-velocity profiles for model with $60^{\circ}$ flap and slat-on at NPR $=1.37$ $\left(M_{\infty}=0.143, \alpha=10^{\circ} ; X=0.99,1.08,1.16\right.$, and 1.24; $Y=-0.42,-0.57$, and -0.65$)$. (a) Uvelocity profiles; (b) V-velocity profiles; and (c) W-velocity profiles. 

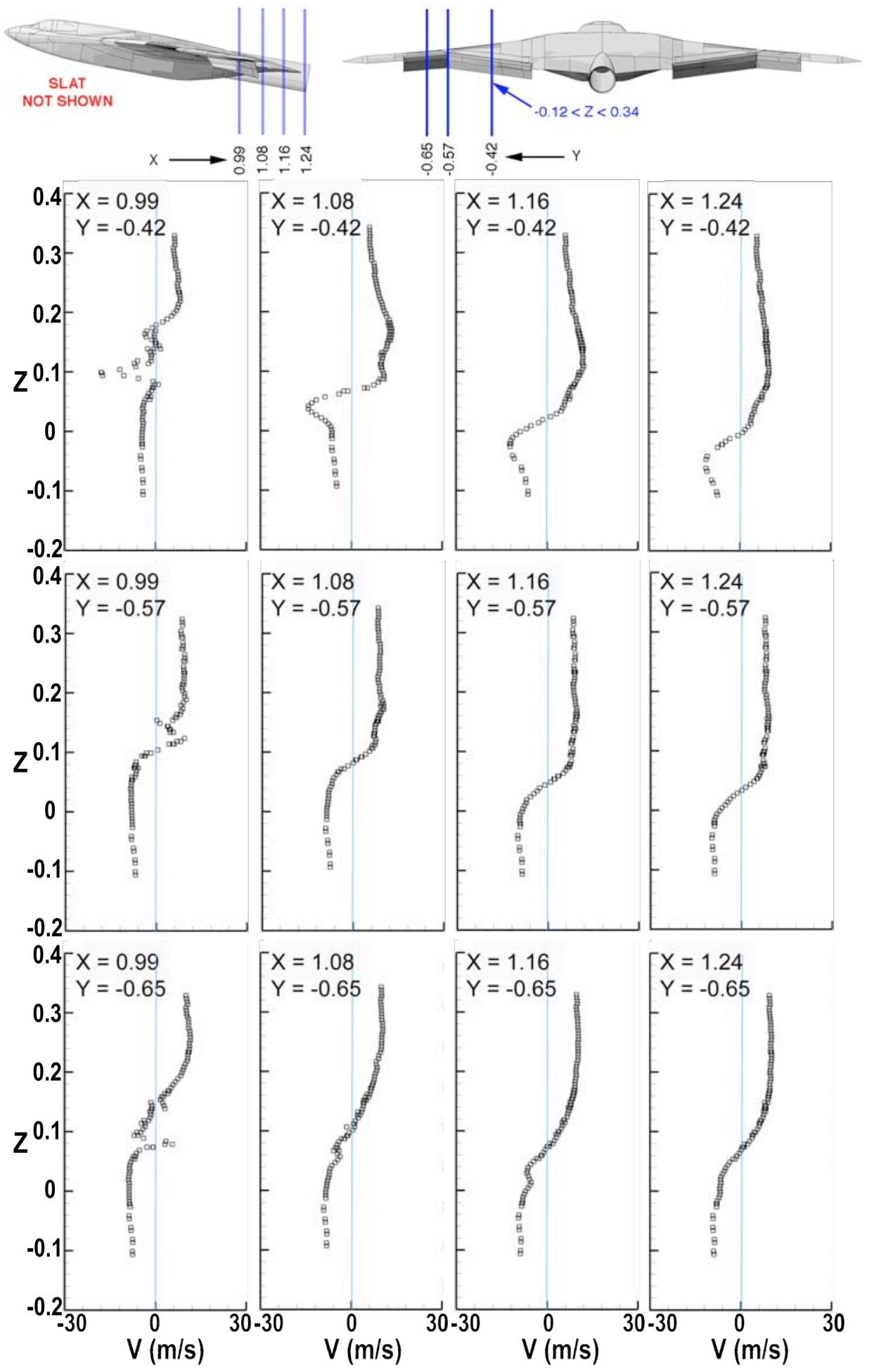

(b) V-velocity profiles (+ represents inward flow, - represents outward flow)

Figure 7. Continued (NPR = 1.37). 


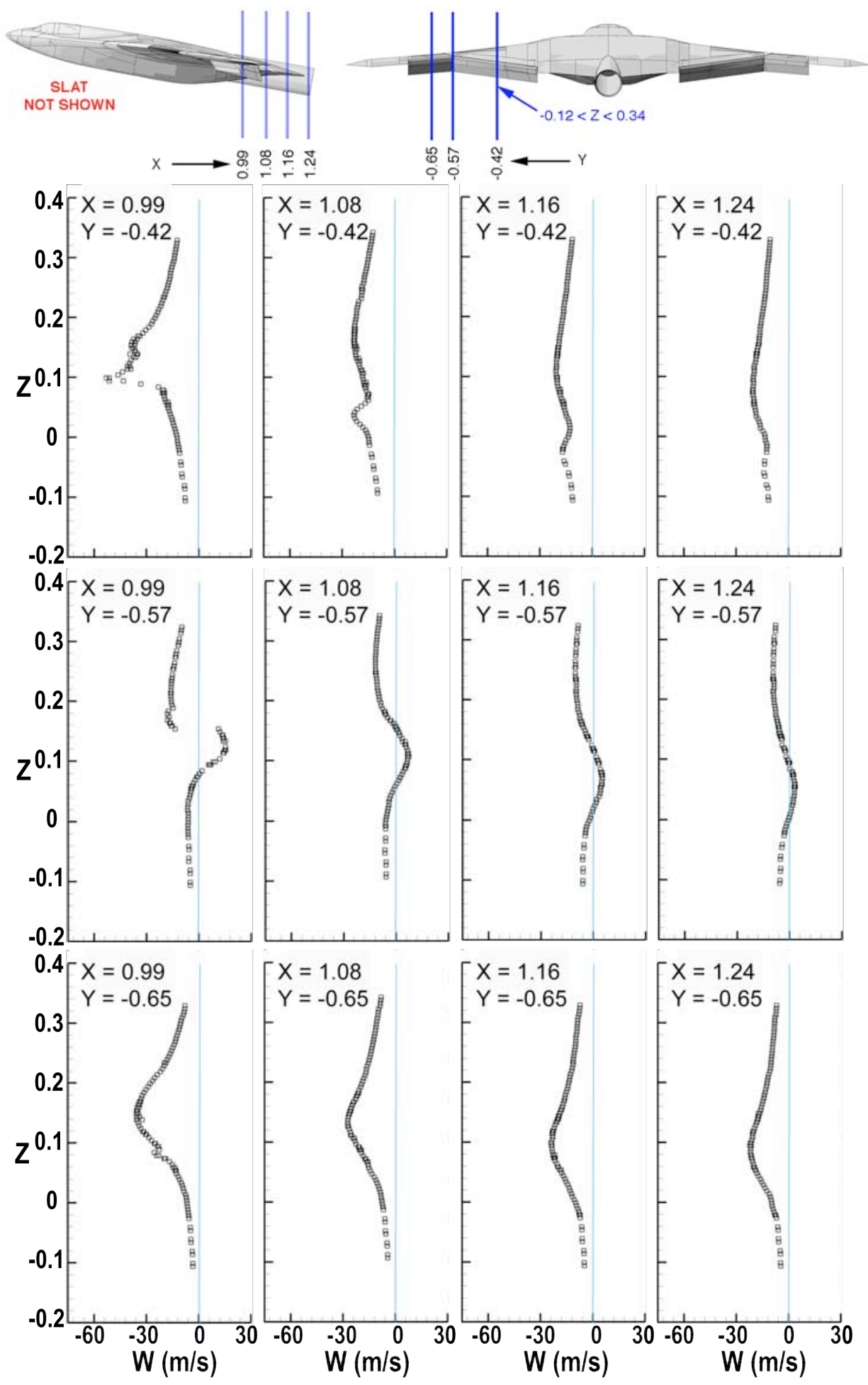

(c) W-velocity profiles (+ represents upward flow, - represents downward flow)

Figure 7. Concluded (NPR = 1.37). 


\subsection{Case B: $0^{\circ}$ Flap without Slat at $\alpha=0^{\circ}$}

After measuring the flow field of the $60^{\circ}$ flap and baseline slat configuration, the slat was removed from the model and the trailing-edge flaps were set to $0^{\circ}$ deflection. Taking the flow-field data without the slat simplified the model geometry and removed the need to simulate the complex flows in the slat region. This isolated the flap-blowing effects for code validation/development. The velocity vectors in the Y-Z plane at X $=1.26$ are shown in Fig. 8 for NPR $=1.0,1.37$, and 1.56 cases at $\alpha=0^{\circ}$, where $\mathrm{X}=1.26$ approximately corresponds to the trailing edge of the model fuselage (see the left sketch on top of Fig. 9).

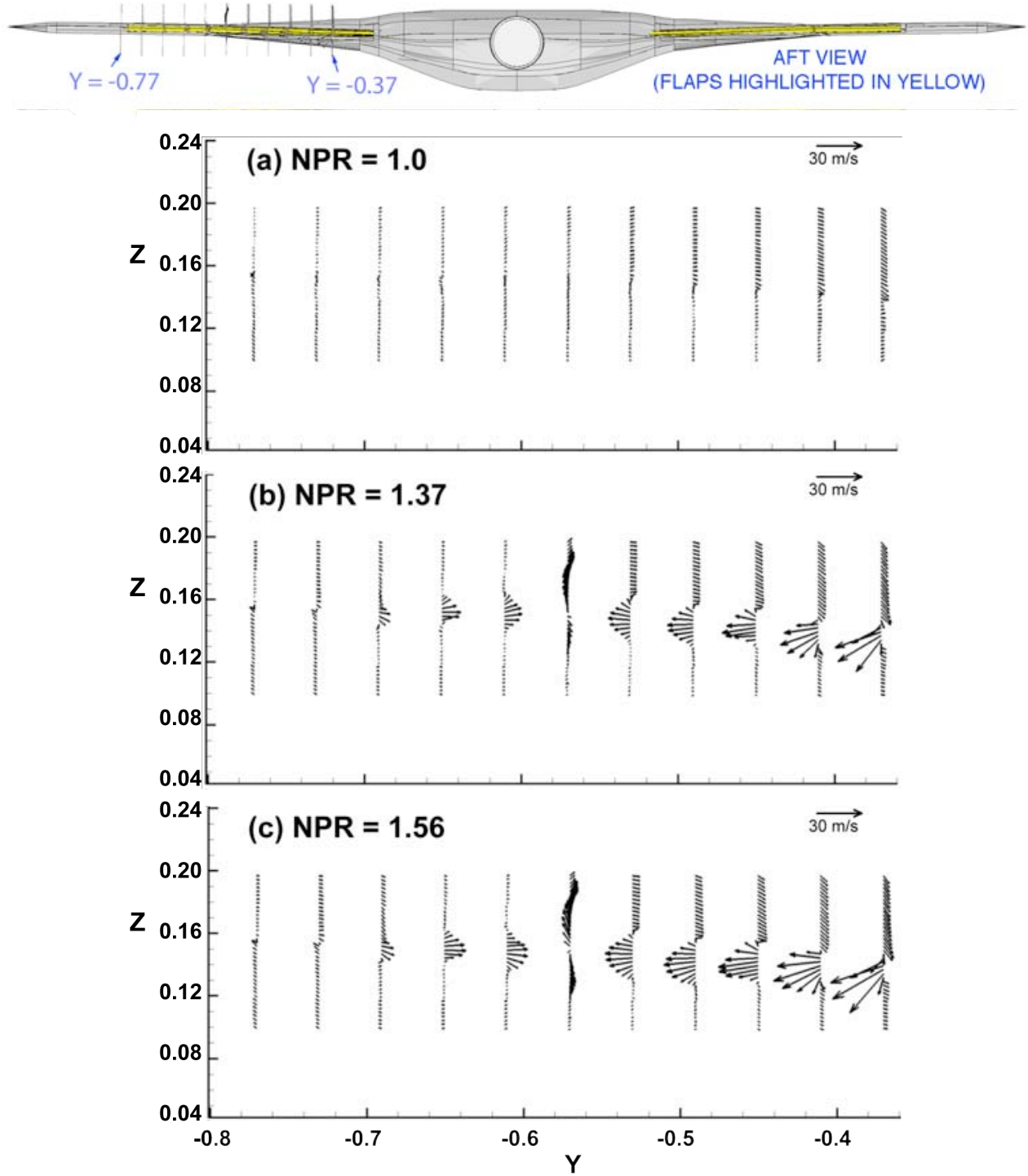

Figure 8. Velocity vectors in $Y-Z$ plane for $0^{\circ}$ flap without slat $\left(\mathrm{M}_{\infty}=0.143, \alpha=0^{\circ}, \mathrm{X}=1.26\right)$. (a) NPR = 1.0; (b) NPR = 1.37; and (c) NPR = 1.56.

Again, the sketch at the top of the figure shows the spanwise locations and the extent of coverage in the vertical direction of survey stations with respect to the model. As expected, Fig. 8 (a) shows relatively small velocity components in the $\mathrm{Y}-\mathrm{Z}$ plane for the non-blowing case (NPR $=1)$. However, for both blowing cases, large velocity components are observed in the Y-direction, and to a lesser 
extent Z-direction. The NPR $=1.56$ case shows similar but slightly stronger velocity vectors than the NPR $=1.37$ case at most stations as expected (see Figs. 8 (b) and 8 (c)). The jet effects are larger at the inboard flap stations in part because of the increased slot height and therefore increased blowing momentum, but also because the probe moved closer to the blowing slot as the survey moved inboard.

Figure 9 shows the U-, V-, and W-velocity profiles (top, middle, and bottom rows, respectively) for the NPR $=1.37$ case at eight selected Y stations. For all stations, Fig. 9 (a) shows not only little or no U-velocity deficit but a significant increase in the local U-velocity (almost 1.6 times the freestream value) due to momentum addition from the blowing jets. The theoretical jet velocity magnitude (Vjet) at the slot exit is $225 \mathrm{~m} / \mathrm{s}\left(\mathrm{Vjet} / \mathrm{U}_{\infty} \approx 4.6\right)$.
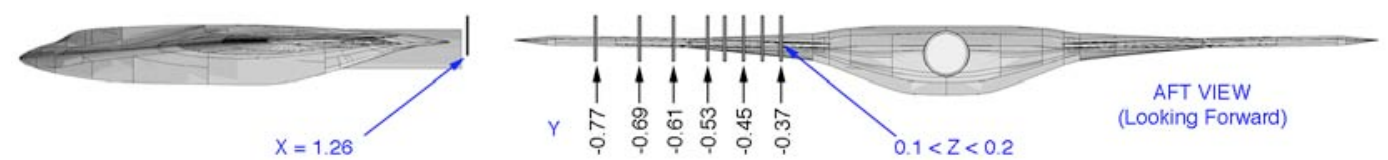

(a) U-velocity profiles

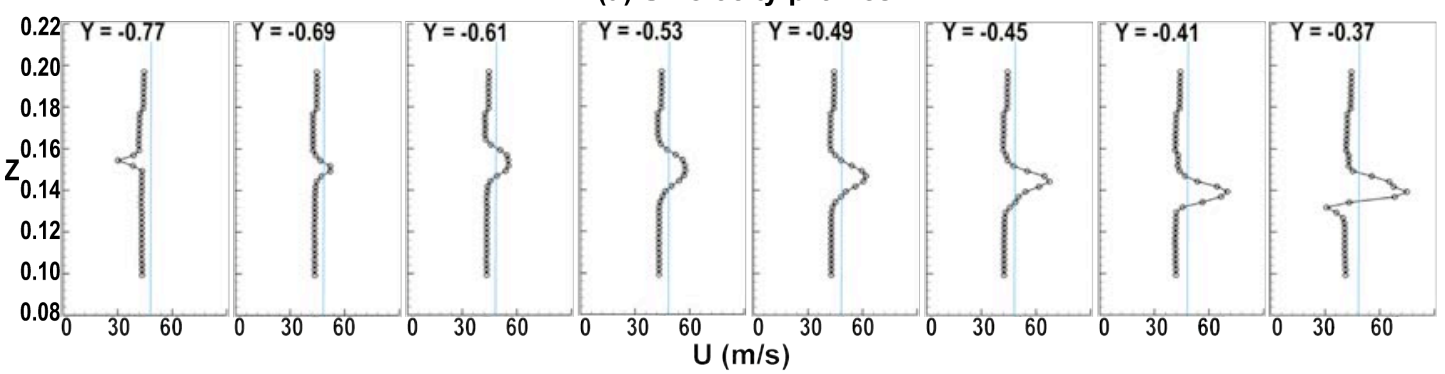

(b) V-velocity profiles

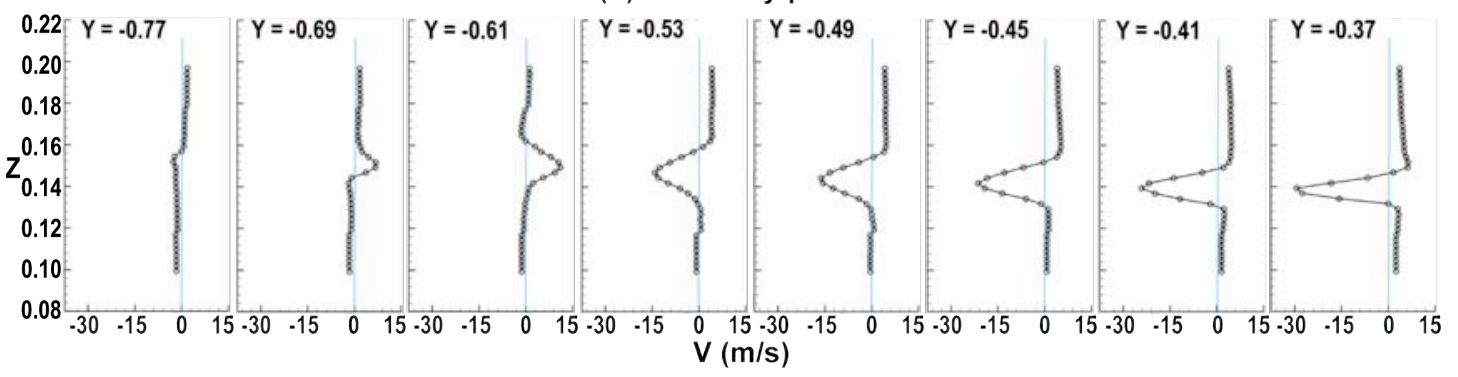

(c) W-velocity profiles

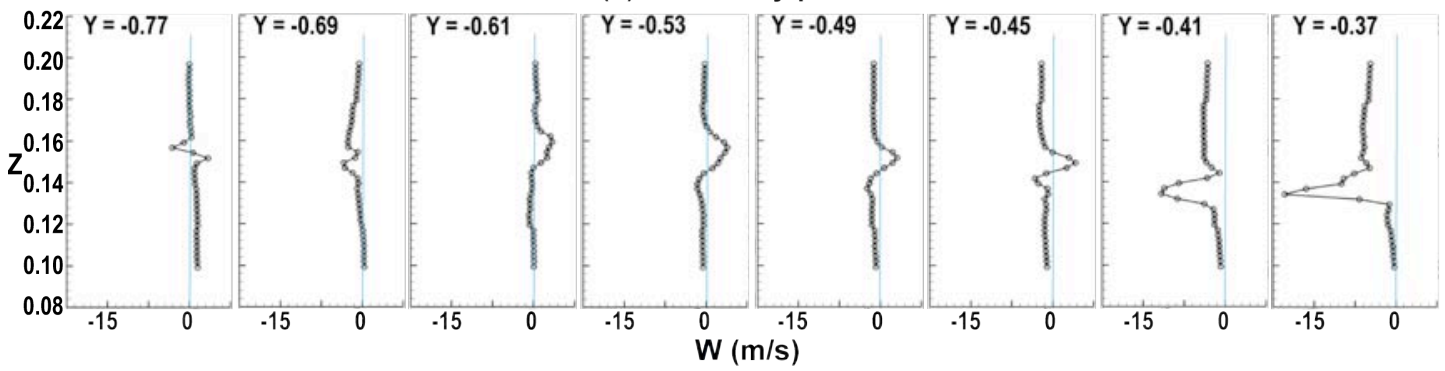

Figure 9. U-, V-, \& $\mathrm{W}$-velocity profiles for $0^{\circ}$ flap without slat at various $\mathrm{Y}$ stations $\left(\mathrm{M}_{\infty}=\right.$ $0.143, \alpha=0^{\circ}, X=1.26, N P R=1.37$ ). (a) U-velocity profiles; (b) $V$-velocity profiles; and (c) $\mathrm{W}$-velocity profiles.

The V-velocity profiles in Fig. 9 (b) show inward flow for the two $\mathrm{Y}$ stations $(\mathrm{Y}=-0.61$ and 0.69 ) downstream of the outboard flap and outward flow for the five $Y$ stations downstream of the inboard flap $(\mathrm{Y}=-0.37,-0.41,-0.45,-0.49$, and -0.53$)$. This was expected because the internal 
blowing plenum is designed to supply flow perpendicular to the wing trailing edge. Because of the swept trailing edges, the V-velocity should be approximately $\mathrm{V}=\mathrm{Vjet} \mathrm{x} \sin \left(\Lambda_{\mathrm{TE}}\right)$, where $\Lambda_{\mathrm{TE}}$ is the corresponding trailing-edge sweep angle that is negative for the inboard flap and positive for the outboard flap. The W-velocity profiles in Fig. 9 (c) show a small up-wash region near the middle portion of the survey $(\mathrm{Z} \approx 0.14$ to 0.16 ) for most cases, except for the two most inboard $\mathrm{Y}$ stations $(\mathrm{Y}=$ -0.37 and -0.41 ) where the jet-induced downwash flows are most dominant.

As previously mentioned, the jet effects are the largest at the most inboard station for all U-, V-, and $\mathrm{W}$-velocity profiles because of the increasing slot height and decreasing distance to the surveyplane as the trailing edge sweeps aft. There was no blowing upstream of the most outboard station (Y $=-0.77$ ), so the minor U-velocity deficit observed there is associated with a small wake from the outboard wing.

\subsection{Case C: $60^{\circ}$ Flap without Slat at $\alpha=0^{\circ}$}

The final case examined was the $60^{\circ}$ flap deflection case without the slat. The velocity vectors in the $\mathrm{Y}-\mathrm{Z}$ plane at $\mathrm{X}=1.26$ for the two blowing cases (NPR $=1.37$ and 1.56 ) at $\alpha=0^{\circ}$ are shown in Fig. 10. No 7-hole probe survey was conduced for the non-blowing case because of expected flow separation on the flap. A courser survey grid was used for the NPR $=1.37$ case because of time constraints.

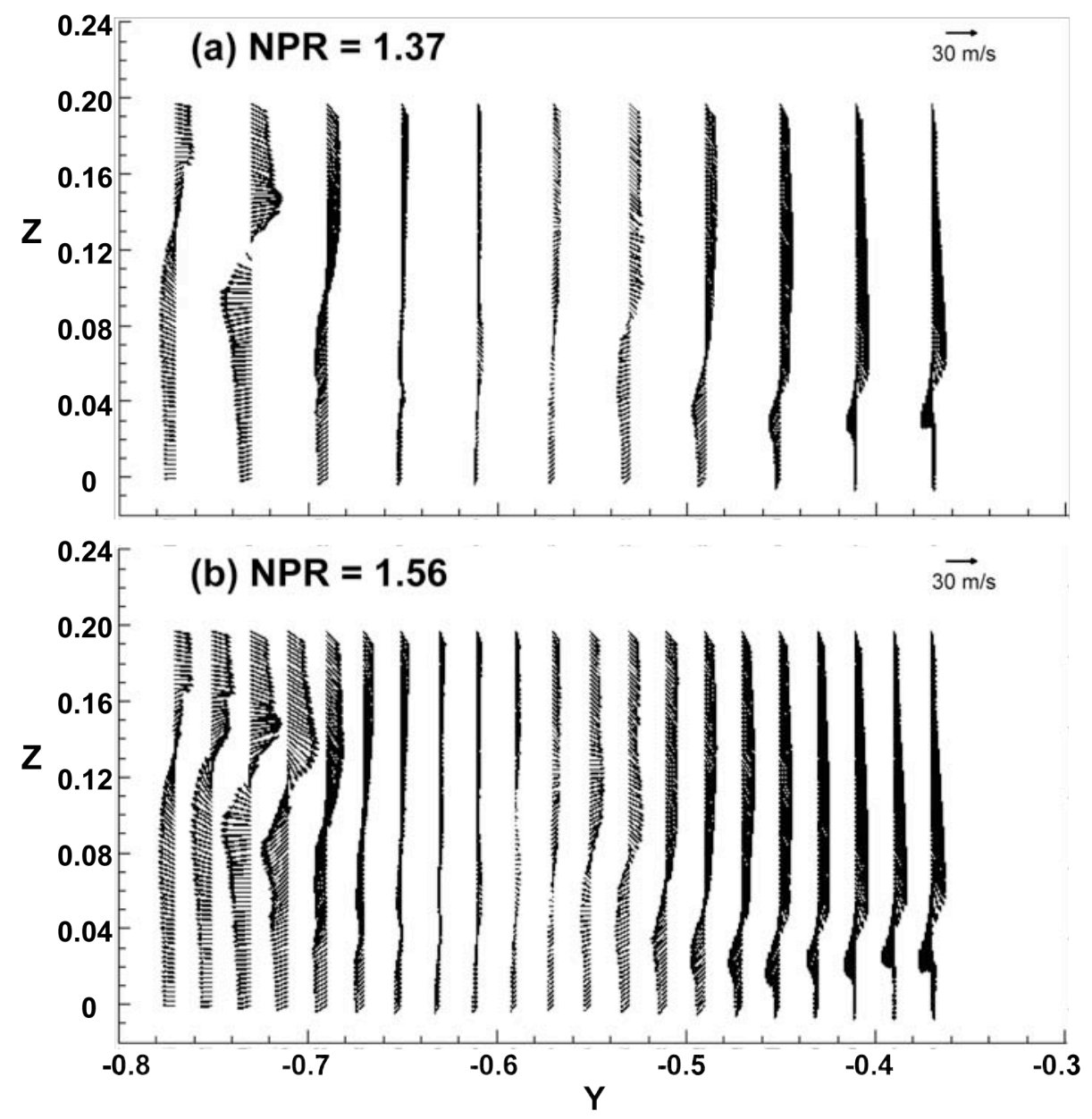

Figure 10. Velocity vectors in $Y-Z$ plane for $60^{\circ}$ flap without slat $\left(M_{\infty}=0.143, \alpha=0^{\circ}, X=\right.$ 1.26). (a) NPR = 1.37; and (b) NPR $=1.56$. 
Figures 10 (a) and 10 (b) show the same general trend between the two blowing cases, where there appears at least two swirling regions similar to those of Case A in terms of the vortex magnitude, location, and direction of rotation.

Figures 11 (a), 11 (b), and 11 (c) show the U-, V-, and W-velocity profiles, respectively, for the $\mathrm{NPR}=1.37$ case at eight selected $\mathrm{Y}$ stations (see sketches at the top of the figure). No significant or obvious jet was observed for the U-, V-, and W-velocity profiles at the four most outboard Y stations. This is consistent with the data at $X=1.24$, as shown in Fig. 7. Most likely the jet has mixed with the local flow at this point and eliminated the jet-induced shear layer.
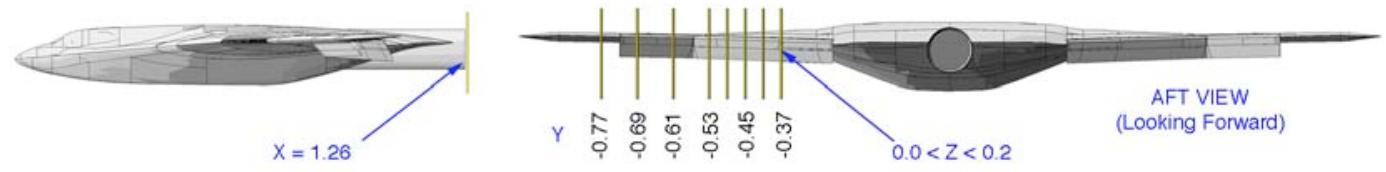

(a) U-velocity profiles

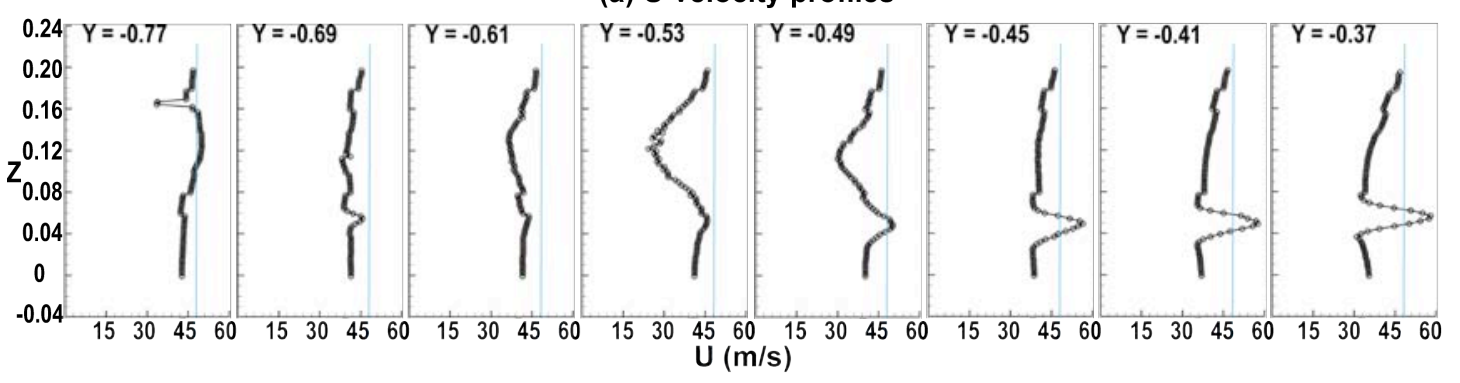

(b) V-velocity profiles

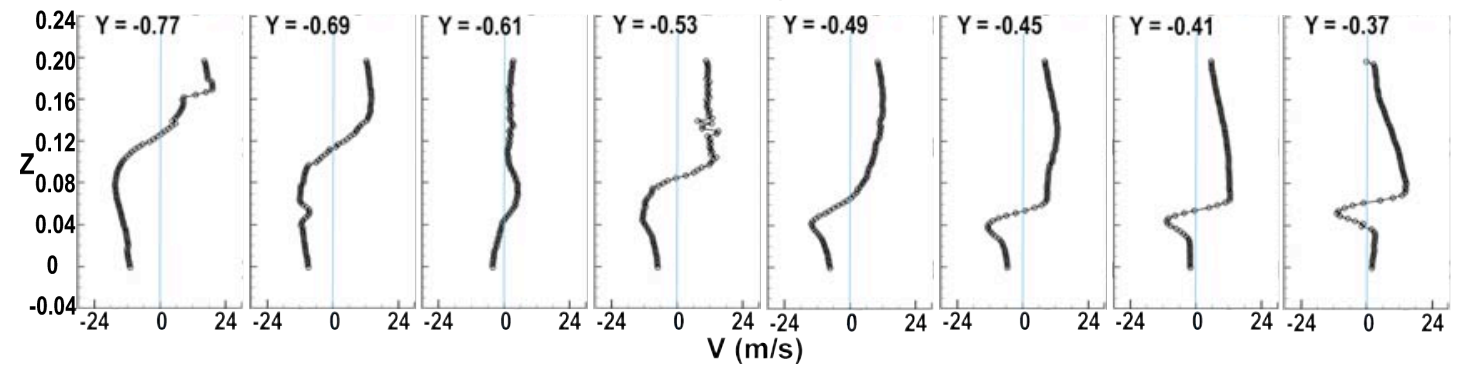

(c) W-velocity profiles

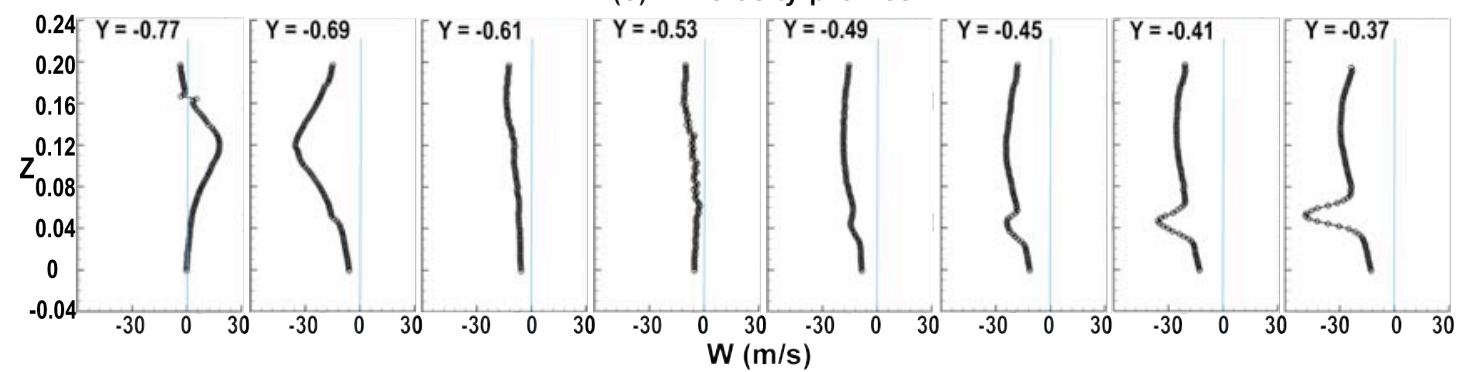

Figure 11. U-, V-, \& $\mathrm{W}$-velocity profiles for $60^{\circ}$ flap without slat at various $\mathrm{Y}$ stations $\left(\mathrm{M}_{\infty}=\right.$ $0.143, \alpha=0^{\circ}, X=1.26, N P R=1.37$ ). (a) U-velocity profiles; (b) $V$-velocity profiles; and (c) W-velocity profiles.

For the four most inboard $\mathrm{Y}$ stations $(\mathrm{Y}=-0.37,-0.41,-0.45$, and -0.49$)$, Fig. 11 shows a continuing increase in the jet-induced U-, V-, and W-velocities, as the survey moved inboard. The Uvelocity increased to almost 25 percent over the freestream and the $\mathrm{W}$-velocity downwash approached the freestream value at the most inboard station $(\mathrm{Y}=-0.37)$. Again, the minor $\mathrm{U}$-velocity deficit 
observed at $\mathrm{Y}=-0.77$ is associated with a small wake from the outboard wing station that was outside of the blowing coverage.

\section{CONCLUDING REMARKS}

Wake surveys using 7-hole probes were successfully performed on a full-span Hybrid Wing Body model with internally blown flaps at the NASA Langley 14 x 22 Foot Subsonic Tunnel. Three model configurations $\left(60^{\circ}\right.$ flap deflection with a slat at $\alpha=10^{\circ}, 0^{\circ}$ flap deflection at $\alpha=0^{\circ}$, and $60^{\circ}$ flap deflection at $\left.\alpha=0^{\circ}\right)$ and three NPR settings (1.0, 1.37, and 1.56) were investigated. The 7-hole probe results further quantified two known swirling regions (downstream of the outboard flap edge and downstream of the inboard/outboard flap juncture) for the $60^{\circ}$ flap cases with blowing. Flow-field results and the general trends are very similar for the two blowing cases of NPR $=1.37$ and 1.56. High downwash velocities corresponded to the enhanced lift for the $60^{\circ}$ flap cases with blowing. Jetinduced effects are the largest at the most inboard station for all three-velocity profiles because of the increasing slot height (and thereby mass flow) and decreasing in distance to the survey-plane as the trailing edge sweeps aft. The results also indicate a limitation of the measurement technique (i.e., flow angles $>75^{\circ}$ with respect to the probe axis). Lessons learned from the current study can be used to improve the future testing of similar models. The experimental data are being used to develop and validate CFD tools for high-lift wings with advanced powered-lift technologies.

\section{ACKNOWLEDGMENTS}

The authors extend their special thanks to the staff and crews of the NASA Langley Research Center 14- by 22-Foot Subsonic Tunnel and the AFRL/Northrop Grumman/NASA research team members for

their contributions during this project.

\section{REFERENCES}

1. Northrop, J. K, "The Development of All-Wing Aircraft," presented at the 35th Wilbur Wright Memorial Lecture, May 1947.

2. Lieback, R. H, "Design of the Blended Wing Body Subsonic Transport," Journal of Aircraft, Vol. 41, No. 1, 2004, pp. 10-25.

3. Collins, S. W., Westra, B. W., Lin, J. C., Jones, G. S., and Zeune, C. H., "Wind Tunnel Testing of Powered Lift, All-Wing STOL Model," RAeS-566-5A3, The Aeronautical Journal, February 2009, pp. 129-137.

4. Jones, G. S., Lin, J. C., Allan, B. G., Milholen, W. E., Rumsey, C. L., and Swanson, R. C., "Overview of NASA's CFD Validation Experiments for Circulation Control Application," RAeS566-8A1, presented at the 2008 International Powered Lift Conference, London, UK, July 2008.

5. Guide for the Verification and Validation of Computational Fluid Dynamics Simulations, AIAA G077-1998 (2002).

6. Neuhart, D. H., and McGinley, C. B., "Free-Stream Turbulence Intensity in the Langley 14- by 22-Foot Subsonic Tunnel," NASA TP-2004-213247, 2004.

7. Johansen, E. S., Rediniotis, O. K., Jones, G. S., "The Compressible Calibration of Miniature Multi-Hole Probes," Journal of Fluids Engineering, Vol. 123, No. 3, 2001, pp. 128-138.

8. Rediniotis, O. K., Hoang, N. T., and Telionis, D. P., "The Seven-Hole Probe: Its Calibration and Use," Forum on Instructional Fluid Dynamics Experiments, Vol. 152, 1993, pp. 21-26.

9. Zeiger, M. D. and Schaeffler, N. W., "Correcting Multi-Hole Probe Alignment Bias Errors PostCalibration," AIAA Paper 2001-0900, presented at the 39th AIAA Aerospace Sciences Meeting \& Exhibit, Reno, NV, January 2001.

10. Epstein, R. J., Carbonaro, M. C., Caudron, F., "Experimental Investigation of the Flowfield About an Upswept Afterbody," Journal of Aircraft, Vol. 31, No. 6, 1994, pp. 1281-1290.

11. Everett, K. N., Gerner, A. A., and Durston, D. A., "Seven-Hole Cone Probes for High Angle Flow Measurement: Theory and Calibration," AIAA Journal, Vol. 21, No. 7, 1983, pp. 992-998.

12. Zilliac, G. G., "Calibration of Seven-Hole Probe for Use in Fluid Flows with Large Angularity," NASA TM 102200, 1989. 